This is the post peer-review accepted manuscript of:

D. Balsamo, G. Gallo, D. Brunelli and L. Benini, "Non-intrusive Zigbee power meter for load monitoring in smart buildings," 2015 IEEE Sensors Applications Symposium (SAS), Zadar, 2015, pp. 1-6.

The published version is available online at:

https://doi.org/10.1109/SAS.2015.7133611

(C)2015 IEEE. Personal use of this material is permitted. Permission from IEEE must be obtained for all other uses, in any current or future media, including reprinting/republishing this material for advertising or promotional purposes, creating new collective works, for resale or redistribution to servers or lists, or reuse of any copyrighted component of this work in other works. 


\section{Non-intrusive Zigbee Power Meter for load monitoring in Smart Buildings}

\author{
Domenico Balsamo and Gianluca Gallo \\ DEI, University of Bologna \\ viale Risorgimento 2, Bologna, Italy \\ \{domenico.balsamo,gianluca.gallo\}@unibo.it
}

\author{
Davide Brunelli \\ University of Trento \\ via Sommarive 9, Trento, Italy \\ davide.brunelli@unitn.it
}

\author{
Luca Benini \\ University of Bologna \\ viale Risorgimento 2, Bologna, Italy \\ luca.benini@unibo.it
}

\begin{abstract}
Energy efficiency in smart buildings requires distributed sensing infrastructure to monitor the power consumption of appliances, machines and lighting sources. The analysis of current and voltage waveforms is fundamental for gathering diagnostic information about the power quality and for reducing power wastage. Moreover, it enables Non-intrusive Load Monitoring (NILM), which is the process of disaggregating a household's total electricity consumption into its contributing appliances, by analysing the voltage and current changes. In this paper, an innovative full Energy-neutral (i.e. battery free) and Non-intrusive Wireless Energy Meter (NIWEM) is presented to measure current, voltage and power factor. As key features, the NIWEM is completely non-invasive and it can self-sustain its operations by harvesting energy from the monitored load. It also features a standard (Zigbee) wireless interface for communication with the smart-building system. Experimental results have confirmed that complete energy sustainability can be achieved also with very low-power loads.
\end{abstract}

Index Terms-Wireless sensor networks, Smart Metering, Energy Harvesting.

\section{INTRODUCTION}

An enormous research effort of the worldwide utility companies is oriented to monitor the energy usage for reducing power consumption, by using smart power meters for industrial, commercial and domestic scenarios. The main aim of smart power metering is to make users aware of the energy consumption in daily activities and then to promote energy saving. Standalone meters are now being replaced by distributed sensing infrastructures, which monitor the power consumption of household's appliances, using various communication strategies. Low-cost, robust and easy-to-use power sensing systems are the most suitable devices to monitor in a pervasive way the $50-60 \mathrm{~Hz}$ electricity in smart buildings [1].

A power monitoring system consists of current and voltage sensors and a processing unit, for the analysis of the power consumption (e.g. RMS values, filtering, time and frequency analysis) to assess the power quality and the energy usage. Moreover, smart meters enable disaggregation techniques such as Non-intrusive Load Monitoring (NILM), that is the process of monitoring the households' voltage and current changes to

Luca Benini is also with Department of Information Technology and Electrical Engineering, ETH Zurich, Switzerland understand which appliances have been used, as well as their individual power consumption [2]. Smart meters with NILM technology are mainly used by utility companies to survey the specific usage of electric power in different scenarios and they are considered a low-cost solution to observe the state of appliances [3].

Traditional energy meters are usually powered through the high-voltage AC line, while Wireless Sensor Nodes (WSNs) are often battery-powered and placed where it is not possible to replace batteries or to exploit the mains. Energy harvesting solutions should be adopted when batteries replacement becomes an expensive overhead.

In this paper, an innovative Energy-Neutral and Nonintrusive Wireless Energy Meter (NIWEM) is presented, with self-power capability for smart metering applications. It embeds a clamp-on current transformer, which is used for the current consumption measurement and energy harvesting. The current transformer is easy-to-install because it does not require any breaking of the high-voltage AC line. The voltage is measured using an innovative non-invasive voltage sensor, which exploits capacitive-coupling elements. This new approach does not require any electrical contact with the mains, which makes the measurement much safer.

As key features, the sensor nodes equip an energy harvesting subsystem, which scavenges energy from the monitored load, making them fully-autonomous. Moreover, the system guarantees the start-up operations without any back-up energy buffer. The NIWEMs feature a low-power, 32-bit NXP microcontroller for data processing and a wireless transceiver for sending data to an Energy@ Home [4] compliant Gateway, via ZigBee PRO standard with Home Automation profile.

The paper is organized as follows: Section II presents an overview of the challenges in Smart Metering systems. Section III shows a description of the implementation of the proposed smart sensor node. In Section IV the network protocol is presented. In Section V, the performance evaluation are illustrated, while Section VI concludes the paper.

\section{RELATED WORKS}

Smart meter design is mainly focused on two categories of services. The first one is oriented to monitor high-power 
consumption in energy distribution systems and smart grids while the second one is oriented to monitor the power consumption of household's appliances. Concerning the highpower monitoring, the self-powered system presented in [15] is a wireless power meter designed for measuring current and voltage in distribution lines of smart grids. It exploits two on-air current sensors and an energy harvesting sub-system. Similarly, an efficient powering system, presented in [16], uses a split core current transformer as energy harvester. The collected energy is used to provide constant output voltage and the excess energy is stored in a back-up battery or supercapacitor. In contrast to grid-oriented systems, the majority of smart meters are designed to monitor the power consumption of single household's appliances. For instance, the wireless meter presented in [17] measures the energy consumption of single appliances and can also switch-off the appliances under control. Similar operations are performed by the wireless nodes presented in [18], which monitor the current consumption with Hall-effect sensors and they are powered through the high-voltage line with an AC/DC converter. In [19] a battery-free energy harvester is implemented on-chip by using $0.25 \mu \mathrm{m}$ CMOS process. It converts the AC signal coming from a Rogowski coil by means of an active rectifier and a set of boost converters combined with a switched capacitor sampler. Another example of meter with energy harvesting capability is given in [20]. It is a miniature device, called energy coupler, capable to deliver up to $10 \mathrm{~mW}$ of power to a $50 \Omega$ load, when placed nearby a conductor of a power distribution system, where the current ranges between $70 \mathrm{~A}$ - 1000A. Finally, two interesting examples of single sensor self-powered meters are given in [21] and in [22]. The first one is based on a novel dual wire current transformer and exploits a wireless monitoring system to perform current measurement and a magnetic energy harvester realized onchip to self-sustain its operations. The second one is a very small current waveform meter, which can harvest energy from the measured current and transmit data over the power line. Commercial devices such as SGM3000 series smart system [7] can contain several meters, for both residential and commercial demand, and they can include single phase and multi-phase metering solutions. Our previous work [6] is related to an IEEE 802.15.4-compliant wireless current meter with energy harvesting capability, which exploits two separate current transformers: one for the measuring and one for harvesting the energy from the main. However, the main drawback of this solution is the lack of an non-intrusive voltage sensing circuit, which is fundamental for a complete measure of the power. Moreover, the proposed solutions are limited in terms of features implemented.

In this paper we will present an efficient dedicated smart energy meters with advanced features. The main idea is to use smart meters to obtain information about energy consumption from household's appliances in order to improve the energy



Fig. 1. Block diagram of the current sensing circuit.

efficiency and the billing. Moreover, it will enable energy disaggregation techniques such as Non Intrusive load monitoring (NILM). The presented meter is compliant to the specifications of Energy@home [4], which is a no-profit association that promotes smart metering and NILM approach. It aims at developing technologies and services for energy efficiency in a smart home, based on device-to-device communication, to create an eco-system of interoperating devices.

\section{SYSTEM DESCRIPTION}

The entire system is composed by wireless energy meters (NIWEM), which measure the power consumption and send data over the ZigBee network to an Energy@Home compliant Gateway that is connected to a java-enabled host. Each meter exploits a clamp-on transformer for current measurement and energy harvesting, and a new non-invasive capacitive-coupling sensor for voltage measurement. The architecture consists of four main blocks: i) the current sensing section, ii) the voltage sensing section, iii) the energy harvester and finally iv) the microcontroller and wireless transceiver.

\section{A. Current Sensing Section}

A common method of converting current to voltage is based on using clamp-on current transformers. These sensors have the obvious benefit of non-intrusive measurement mainly because the low-voltage DC circuit is clearly decoupled from the high-voltage input, which makes this measure much safer. The adopted current transformer features linear performance over a wide range of inputs and it is easy-to-install because it does not need any interruption or breaking of the voltage line. The main electrical characteristics of this sensor are the number of turns $(n=3000)$ and the maximum measured current (60A), which corresponds to an input current of $20 \mathrm{~mA}$.

Fig. 1 shows the block diagram for the current measurement where the current is converted to a proportional voltage, using the I-to-V Converter. The voltage is then sampled using an Analog-to-Digital converter and a 32-bit microcontroller. Moreover, a Raised Cosine Filter (RCF) is implemented to reduce the high-frequency noise. The RCF is a low-pass 
filter, which is commonly used for pulse shaping in data transmission systems. The frequency response $\mathrm{H}(\mathrm{f})$ of this filter is symmetrical and it is divided into three main parts: it is constant in the pass-band, it sinks in a cosine curve to zero through the transition region and it is zero outside the passband. Its frequency-domain description is a piecewise function, given by:

$H(f)=\left\{\begin{aligned} T & |f| \leq \frac{1-\beta}{2 T}, \\ \frac{T}{2}\left[1+\cos \left(\frac{\pi T}{\beta}\left[|f|-\frac{1-\beta}{2 T}\right)\right]\right. & \frac{1-\beta}{2 T}<|f| \leq \frac{1+\beta}{2 T}, \\ 0 & \text { otherwise. }\end{aligned}\right.$

where $\beta$ is the roll-off factor $(0 \leq \beta \leq 1)$, and $\mathrm{T}$ is the reciprocal of the symbol-rate. The response of a real filter is an approximation to this behaviour. The filter is designed as a Finite Impulse Response filter (FIR) $(\mathrm{f}=12.5 \mathrm{KHz}$, corner frequency $500 \mathrm{~Hz},-6 \mathrm{~dB})$.

\section{B. Voltage Sensing Section}

Current consumption measurement, using the magnetic field of a single wire, is a well-established technique. However, there are no non-invasive sensors commercially available for the voltage measurement.

The analysis of the voltage is important for measuring the Power Factor (PF), which is the ratio between the real power $(\mathrm{P})$ measured in $(\mathrm{W})$ and the apparent power $(\mathrm{S})$ measured in (VA). PF can theoretically have values in the range from 0 to 1, but in real-world applications it has to be bigger than 0.9 mainly because the utility companies supply customers with apparent power (S) but the utility bill is related to the real power $(\mathrm{P})$. When the $\mathrm{PF}$ is lower than 1, the companies need to generate more than the minimum volt-amperes needed to supply the real power, increasing the costs for the energy generation and transmission.

The main idea for measuring the voltage with a non-invasive sensor is to use capacitive-coupling elements positioned near to the electric field generated by the mains. These elements can generate a current in response to the voltage variation because they are coupled to the conductive parts of each cable through the external insulation. These capacitive-coupling elements can be modelled using the transfer function $H(j \omega)$ of a first-order electronic high-pass filter given by:

$$
H(j \omega)=\frac{\operatorname{Vout}(j \omega)}{\operatorname{Vin}(j \omega)}=\frac{j \omega R C}{j \omega R C+1}
$$

where $C$ and $R$ are respectively the capacitance of the capacitive-coupling elements and the measuring resistance.

The cut-off frequency $f t$ of this filter is given by:

$$
f(t)=\frac{1}{\pi R C}
$$

Fig. 2 shows the two capacitive-coupling elements and the block diagram for the voltage measurement. In the electrical

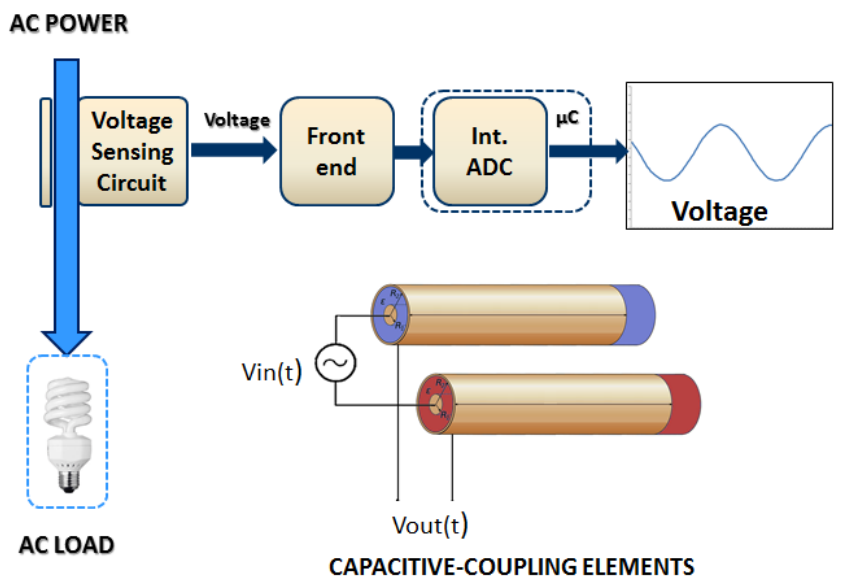

Fig. 2. Block diagram of the voltage sensing circuit.

distribution systems the voltage is measured between two conductors (e.g. phase $\mathrm{P}$ and neutral N). However, these conductors are floating compared to the NIWEM meter, if a common reference is not available. The differential measurement (Fig. 2) allows to eliminate the floating component and then to only obtain the useful voltage, without any common reference to the main high-voltage line.

\section{Energy Harvester}

The energy harvesting circuit uses the same current transformer for the current measurement as power transducer. When the system is in active mode, the transformer is used for sensing current while it is connected to the energy harvesting sub-system, which recovers the energy used in active mode, when the node is in sleep mode. Fig. 3 shows the block diagram of the proposed sub-system, which is used to switch from the current measurement circuit to the energy harvesting sub-system. The switching systems has been already discussed here [13], [14].

NIWEM is able to guarantee the start-up reliably without using any external energy source (e.g. batteries) because it equips on-board a supercapacitor to buffer the harvested energy. The system starts executing the association with the Energy@Home compliant gateway only when the supercapacitor has enough charge. Section V will deeply discuss the curve for the minimum start-up time to guarantee a proper association and the sustainable curve for the minimum sleep time as a function of the monitored load.

\section{Microcontroller and Wireless Transceiver}

The smart power meter features the JN5148 module from NXP, which is a low-power and high-performance 32-bit microcontroller (MCU) with an integrated $2.4 \mathrm{GHz}$ IEEE 802.15.4/ZigBee PRO radio transceiver. Furthermore, the MCU provides a $512 \mathrm{kB}$ serial flash memory, a real-time clock, an integrated antenna, a $128 \mathrm{kB}$ ROM, a $128 \mathrm{kB}$ RAM, a 


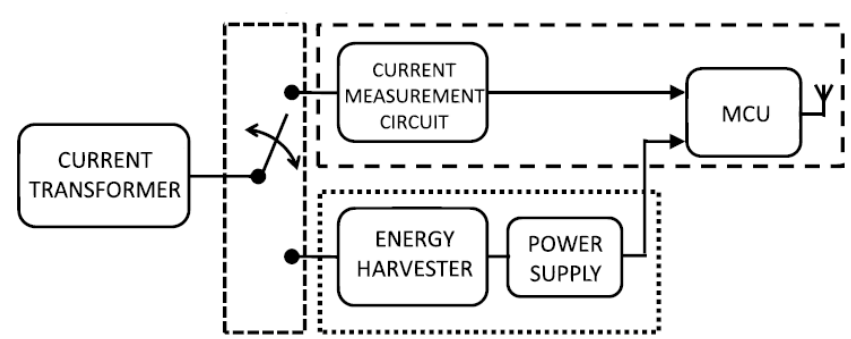

Fig. 3. Block diagram of the switch system



Fig. 4. Contact-less Smart power meter prototype.

set of analogue and digital peripherals (12-bit ADC and 12bit DAC) and, finally, SPI and I2C interfaces. This module is characterized by a very low-power consumption in sleep mode (only $2.6 \mathrm{uA}$ ) while, in active mode, the current consumption is dominated by the radio transceiver, which requires $15 \mathrm{~mA}$ for transmit and $17.5 \mathrm{~mA}$ for receive. All the values are referred to as a supply voltage of $3.0 \mathrm{~V}$, that is the normal operating voltage of this wireless energy meter.

The smart energy meter transmits data to the Energy@Home compliant Gateway, using ZigBee PRO protocol with Home Automation profile. This gateway is a commercial product called FlexKey and it is one of the most compact Zigbee USB Dongle available on the market today ${ }^{1}$. Due to its RF performance, it enables secure and reliable wireless connectivity between internal and external environment. The Gateway has been designed to be connected to every device equipped with a USB port (ADSL modem, PC, Tablet). Fig. 4 shows the prototype of the smart power meter and the Energy@Home compliant Gateway.

\section{Network Protocol And Architecture}

The FlexKey USB Gateway acts as a ZigBee PRO coordinator with Home Automation profile. ZigBee is a wireless

\footnotetext{
${ }^{1}$ http://www.flexgrid.it/eng/prodotti/flexkey.html
}

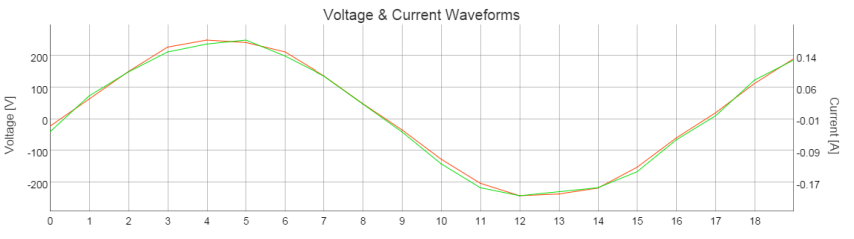

Last data: $10 \mathrm{sec}$ ago

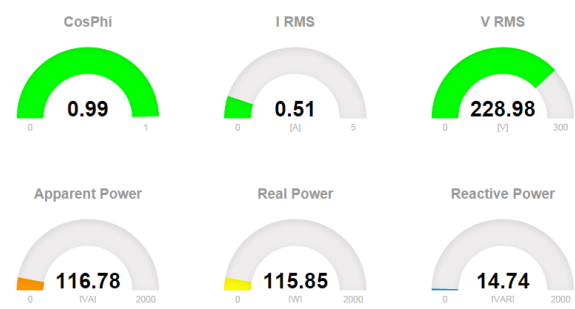

Fig. 5. Application data view

networking technology developed by ZigBee Alliance for lowdata rate and short-range applications.

ZigBee protocol consists of four main layers: physical (PHY), medium access control (MAC), network (NWK) and application (APL). In addition, ZigBee provides security functionality across layers. The bottom layers of ZigBee stack are defined by the IEEE 802.15.4 standard, while the top of this stack is defined by the ZigBee Alliance specification. ZigBee PRO Home Automation is a new profile for smart buildings, that enhance comfort, convenience, security and energy management for the consumer and it adds several features, which improve the life-time of WSNs and simplify installation and maintenance for consumers and custom installers.

NIWEM with ZigBee PRO Home Automation profile samples the current and voltage waveforms and provides the following parameters: RMS current, RMS voltage and power factor. These data are then transmitted through the network. The total amount of data sent is equal to 94 byte. These data are transmitted using two packets: the first one contains 60 byte of payload, where 40 byte are for the current waveform and 20 byte for the voltage waveform, while the second one contains the remaining 20 byte of the voltage waveform and the RMS current ( 2 byte), the RMS voltage ( 2 byte), the MCU supply ( 2 byte) and the power factor ( 4 byte). The use of a multi-packet solution is because the payload length can not exceed 60 byte due to the configuration of the network with this specific Gateway. To mitigate the fragmentation and to reduce the sending rate, data compression techniques, such as Compressive Sensing (CS) [11] will be verified in future.

Moreover, we have decided to not use the ZigBee PRO automatic fragmentation and to perform a high-level check for the reception of these packets.

On the host-side, we have implemented a Java multiplatform software (WiStation), which is able to run with every 


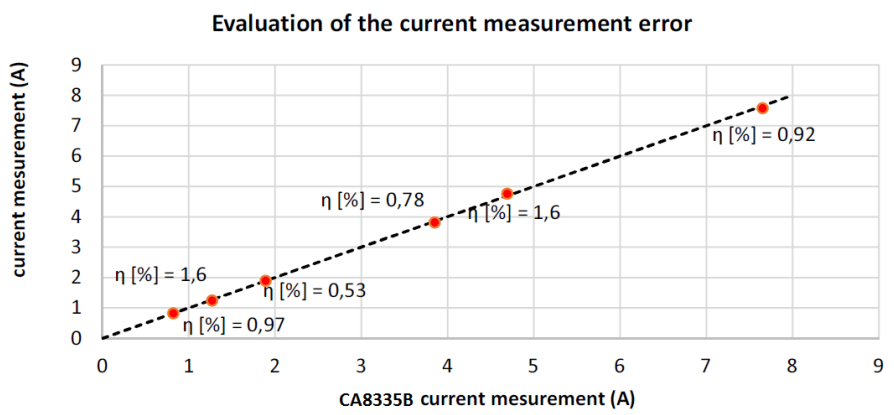

Fig. 6. Evaluation of current measurement error

machine (e.g. Windows, Linux, Beaglebone, Raspberry Pi, Android devices) and it acts as a control station. This software communicates with the ZigBee Gateway using an USB virtual serial port and it allows the end-users to setup, connect and configure the ZigBee network architecture. Moreover, it performs packet check and it alerts with specific notification in case of lost packets/nodes. The system displays the information using HTML/JavaScript pages with a local web server, which is embedded into the application. In this way, it is possible to keep the software completely multi-platform and to enable the remote control of the ZigBee network. The main WiStation web data view is shown in Fig. 5. This interface can be used by both administrator and end-users for monitoring the voltage and current waveforms, the RMS values for the current and the voltage, the power factor, the apparent, real and reactive powers.

\section{Performance evaluation}

Performance has been assessed in terms of measurement errors in the current, voltage and power factor measurements and of the energy harvesting capability. Fig. 6 shows a comparison between the current consumption of few appliances measured with both a calibrated power meter (Chauvin Arnoux CA8335B Mains-analysis device) and our NIWEM. It is possible to notice that the maximum error of the smart energy sensor node is less than $2 \%$. This error is mainly due to the harmonic content of the measured current (e.g. high-frequency harmonic distortion). The results and the accuracy achieved are however very good considering that the cost of the proposed system is about 40 times lower than the calibrated power meter.

Moreover, Table I shows the measurement of the current, voltage and power factor for three specific loads: a $500 \mathrm{~W}$ lamp, a resistive-capacitive load and a PC screen. Also in this case, we present a comparison between the consumption of these appliances measured with both a calibrated power meter (CA8335B) and our wireless energy meter. In more detail, the current, the voltage and the power factor are firstly measured using the CA8335B and then the same measurements are performed using the smart wireless energy meter. The values obtained with the presented solution are very close (the error
TABLE I

EVALUATION OF VOLTAGE, CURRENT AND POWER FACTOR MEASUREMENT

\begin{tabular}{|l|c|c|c|}
\hline 500 W lamp & Voltage [V] & Current [A] & Power Factor \\
\hline CA8335B & 224.3 & 1.956 & 1.00 \\
\hline NIWEM & 227.3 & 1.960 & 0.99 \\
\hline
\end{tabular}

\begin{tabular}{|l|c|c|c|}
\hline Res. - Cap. & Voltage [V] & Current [A] & Power Factor \\
\hline CA8335B & 226.5 & 0.688 & 0.53 \\
\hline NIWEM & 227.1 & 0.680 & 0.52 \\
\hline
\end{tabular}

\begin{tabular}{|l|c|c|c|}
\hline PC Monitor & Voltage [V] & Current [A] & Power Factor \\
\hline CA8335B & 225.5 & 0.170 & 0.54 \\
\hline NIWEM & 227.7 & 0.173 & 0.53 \\
\hline
\end{tabular}

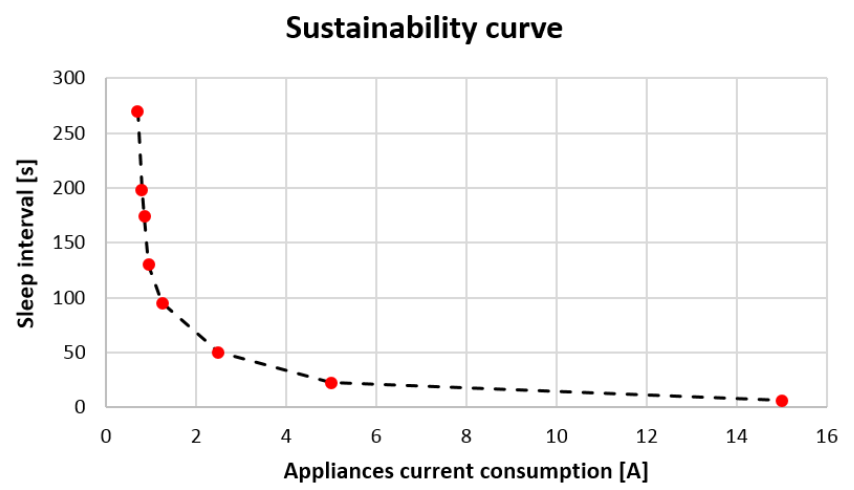

Fig. 7. Sustainability curve. For a given appliance current consumption the curve returns the minimum sleep time interval to guarantee sustainable operations

is negligible) to the values obtained by using Chauvin Arnoux CA8335B.

In Figure 7, we illustrate the curve of the optimal sleep time as a function of the power dissipation of the main appliances. This curve is obtained with experimental data and it shows that every appliance, with a specific current consumption, is characterized by a specific duty-cycle, which permits a periodic measurement, without any external battery.

Moreover, the system can start-up reliably without using any back-up energy buffer. The time for charging the on-board supercapacitor changes according to the input current: appliances with higher power consumption permit the current transformer to deliver higher power, hence the start-up interval is shorter and vice versa. In Figure 8, the graph shows the curve of the time needed for the start-up operations as a function of the input power, with the supercapacitor completely discharged. In particular, the system can have a fast start-up (e.g. with a time less than 60 seconds) if the monitored load is bigger than $100 \mathrm{~W}$. Moreover, the system will be able to properly start-up also with lower power consumption but it will take longer. Of course, the provided power has to be bigger than the quiescent consumption, which are intrinsic in every device. In the experiments, we verified that our prototype can startup autonomously with minimum load of $10 \mathrm{~W}$. 


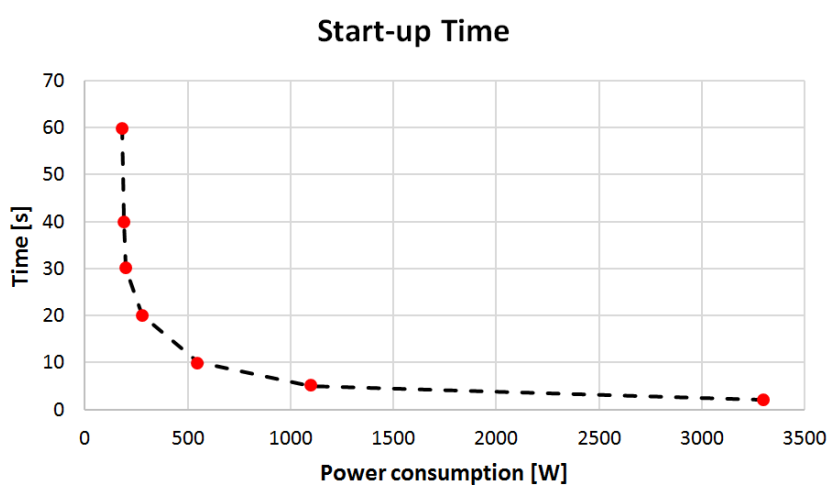

Fig. 8. Start-up curve. For a given appliance power consumption the curve returns the minimum start-up time interval to guarantee a proper association with the Energy@Home Freescale gateway.

\section{CONCLUSIONS}

In this paper, a new Energy-Neutral and Non-intrusive Wireless Energy Meter is presented, for measuring voltage, current and power factor for residential and industrial appliances. As key features, it can self-sustain its operations by harvesting energy from the monitored load. Moreover, this system feature a low-power, 32-bit NXP microcontroller for data processing and a wireless transceiver for sending data, via ZigBee PRO standard with Home Automation profile, to a Energy@Home compliant gateway. Experimental results have confirmed that a complete energy sustainability and an autonomous start-up can be achieved without using any external batteries. Finally, the accuracy of node has been characterized by measuring the current, voltage and power factor of a set of three appliances where the error, with respect to a calibrated instrument, is negligible.

\section{ACKNOWLEDGMENT}

The research contribution presented in this paper has been supported by a research grant from Telecom Italia, by the project GreenDataNet (grant n.609000) funded by the EU 7th Framework Programme and by project FLEXMETER (grant n.646568) funded by the EU H2020 Framework Programme.

\section{REFERENCES}

[1] S. Ahmad, "Smart metering and home automation solutions for the next decade," in Emerging Trends in Networks and Computer Communications (ETNCC), 2011 International Conference on, April 2011, pp. 200204.

[2] J. Liang, S. K. Ng, G. Kendall, and J. W. Cheng, "Load signature study - part 1: Basic concept, structure, and methodology," Power Delivery, IEEE Transactions on, vol. 25, no. 2, pp. 551-560, April 2010.

[3] J. Liang, S. K. Ng, G. Kendall, John W.M., and Cheng, "Load signature study - part 2: Disaggregation framework, simulation, and applications," Power Delivery, IEEE Transactions on, vol. 25, no. 2, pp. 561-569, April 2010.

[4] "Energy@home." [Online]. Available: "http://www.energy-home.it"

[5] J. Zheng, D. Gao, and L. Lin, "Smart meters in smart grid: An overview," in Green Technologies Conference, 2013 IEEE, April 2013, pp. 57-64.
[6] D. Porcarelli, D. Balsamo, D. Brunelli, and G. Paci, "Perpetual and lowcost power meter for monitoring residential and industrial appliances," in Design, Automation Test in Europe Conference Exhibition (DATE), 2013, March 2013, pp. 1155-1160.

[7] "Sgm300 residential meter." [Online]. Available: "https://www. gedigitalenergy.com/smartmetering"

[8] C. Duarte, P. Delmar, K. Goossen, K. Barner, and E. Gomez-Luna, "Non-intrusive load monitoring based on switching voltage transients and wavelet transforms," in Future of Instrumentation International Workshop (FIIW), 2012, Oct 2012, pp. 1-4.

[9] S. Gupta, M. S. Reynolds, and S. N. Patel, "Electrisense: Single-point sensing using emi for electrical event detection and classification in the home," in Proceedings of the 12th ACM International Conference on Ubiquitous Computing, ser. Ubicomp '10. New York, NY, USA: ACM, 2010, pp. 139-148. [Online]. Available: http://doi.acm.org/10.1145/1864349.1864375

[10] S. Drenker and A. Kader, "Nonintrusive monitoring of electric loads," Computer Applications in Power, IEEE, vol. 12, no. 4, pp. 47-51, Oct 1999.

[11] C. Caione, D. Brunelli, and L. Benini, "Compressive sensing optimization for signal ensembles in wsns," Industrial Informatics, IEEE Transactions on, vol. 10, no. 1, pp. 382-392, Feb 2014.

[12] C. Laughman, K. Lee, R. Cox, S. Shaw, S. Leeb, L. Norford, and P. Armstrong, "Power signature analysis," Power and Energy Magazine, IEEE, vol. 1, no. 2, pp. 56-63, Mar 2003.

[13] D. Porcarelli, D. Brunelli, and L. Benini, "Clamp-and-measure forever: A mosfet-based circuit for energy harvesting and measurement targeted for power meters," in Advances in Sensors and Interfaces (IWASI), 2013 5th IEEE International Workshop on, June 2013, pp. 205-210.

[14] D. Porcarelli, D. Brunelli, and L. Benini, "Clamp-and-forget: a self-sustainable non-invasive wireless sensor node for smart metering applications," Microelectronics Journal, vol. 45, no. 12, pp. 1671-1678, Dec. 2014. [Online]. Available: http://www.sciencedirect.com/science/ article/pii/S0026269214001785

[15] F. Cai, E. Farantatos, R. Huang, A.P. Sakis Meliopoulos and J. Papapolymerou, "Self-powered smart meter with synchronized data," in Proc. of the IEEE Radio and Wireless Symposium (RWS 2012), Jan 15-18 2012, pp. 395-398.

[16] C. Joseph, C. William, G. Dmitri, S. Brent, S. Vladimir and G. Michael, "High efficiency powering system for wireless sensor for AC monitoring in smart grid applications," Emerging Technologies for a Smarter World (CEWIT), 2014 11th International Conference \& Expo on , pp. 1-5, 29-30 Oct. 2014.

[17] A. Reinhardt, D. Burkhardt, P. S. Mogre, M. Zaheer and R. Steinmetz, , "Smartmeter.kom: A low-cost wireless sensor for distributed power metering," in IEEE 36th Conference on Local Computer Networks $(L C N)$, 2011, pp. 1032-1039.

[18] X. Jiang, S. Dawson-Haggerty, P. Dutta and D. Culler, "Design and implementation of a high-fidelity ac metering network," in International Conference on Information Processing in Sensor Networks, 2009. IPSN 2009, pp. 253-264.

[19] Tzu-Chi Huang et al., "A battery-free energy harvesting system with the switch capacitor sampler (scs) technique for high power factor in smart meter applications," in Proc. of the 19th IEEE/IFIP international conference on VLSI and System-on-Chip, 2011 (VLSI-SoC 2011), Oct 3-5 2011, pp. 359-362.

[20] R. H. Bhuiyan, R. A. Dougal, M. Ali, "A miniature energy harvesting device for wireless sensors in electric power system," IEEE Sensors Journal, vol. 10, no. 7, pp. 1249-1258, 2010.

[21] T. C. Huang et al., "Non-invasion power monitoring with 120energy improvement by maximum power extracting control for high sustainability power meter system," in IEEE Custom Integrated Circuits Conference (CICC), 2012, pp. 1-4.

[22] S. Takahashi, N. Yoshida, K. Maruhashi, M. Fukaishi, "Real-time current-waveform sensor with plugless energy harvesting from ac power lines for home/building energy-management systems," in IEEE International Solid-State Circuits Conference Digest of Technical Papers (ISSCC), 2011, pp. 220-222. 